\title{
THE
}

1998

\section{Forward projection of transient signals obtained from a fiber-optic pressure sensor}

\author{
G. T. Clement \\ University of Rhode Island \\ R. Liu \\ University of Rhode Island
}

S. V. Letcher

University of Rhode Island

P. R. Stephanishen

University of Rhode Island

Follow this and additional works at: https://digitalcommons.uri.edu/phys_facpubs

Terms of Use

All rights reserved under copyright.

\section{Citation/Publisher Attribution}

Clement, G. T., Liu, R., Letcher, S. V., \& Stephanishen, P. R. (1998). Forward projection of transient signals obtained from a fiber-optic pressure sensor. Journal of the Acoustical Society of America, 104(3), 1266-1273. doi: 10.1121/1.424335.

Available at: https://doi.org/10.1121/1.424335

This Article is brought to you for free and open access by the Physics at DigitalCommons@URI. It has been accepted for inclusion in Physics Faculty Publications by an authorized administrator of DigitalCommons@URI. For more information, please contact digitalcommons-group@uri.edu. 


\section{Forward projection of transient signals obtained from a fiber-optic pressure sensor}

G. T. Clement, R. Liu, S. V. Letcher, and P. R. Stepanishen

Citation: The Journal of the Acoustical Society of America 104, 1266 (1998); doi: 10.1121/1.424335

View online: https://doi.org/10.1121/1.424335

View Table of Contents: http://asa.scitation.org/toc/jas/104/3

Published by the Acoustical Society of America

\section{Articles you may be interested in}

Forward and backward projection of acoustic fields using FFT methods

The Journal of the Acoustical Society of America 71, 803 (1982); 10.1121/1.387606

A wave vector, time-domain method of forward projecting time-dependent pressure fields

The Journal of the Acoustical Society of America 90, 2782 (1991); 10.1121/1.401875

Wave-number domain separation of the incident and scattered sound field in Cartesian and cylindrical coordinates

The Journal of the Acoustical Society of America 97, 2293 (1995); 10.1121/1.411954

Time-dependent multipoles and their application for radiation from volume source distributions Journal of Mathematical Physics 37, 682 (1996); 10.1063/1.531408

Nearfield acoustic holography: I. Theory of generalized holography and the development of NAH The Journal of the Acoustical Society of America 78, 1395 (1985); 10.1121/1.392911

Time domain holography: Forward projection of simulated and measured sound pressure fields The Journal of the Acoustical Society of America 116, 142 (2004); 10.1121/1.1763600 


\title{
Forward projection of transient signals obtained from a fiber- optic pressure sensor
}

\author{
G. T. Clement, R. Liu, and S. V. Letcher \\ Department of Physics, University of Rhode Island, Kingston, Rhode Island 02881 \\ P. R. Stepanishen \\ Department of Ocean Engineering, University of Rhode Island, Kingston, Rhode Island 02881
}

(Received 10 December 1996; accepted for publication 4 June 1998)

\begin{abstract}
An analytical/experimental approach is presented to reconstruct the space-time pressure field in a plane and forward project the resultant space-time pressure field using tomographic and wave vector time-domain methods. Transient pressure signals from an underwater ultrasonic planar transducer are first measured using a line fiber-optic pressure sensor which is scanned across a plane at a fixed distance $z_{0}$ from the transducer. The resulting spatial line integrals in the plane are time-dependent signals which are first used to reconstruct the space-time pressure field in the plane via simply implemented tomographic methods. These signals are then used to forward project the space-time pressure field to arbitrary planes employing a wave vector time-domain method. Verification of the method is first presented using synthetic signals and the impulse response approach. An experimental verification of the approach is then presented using an ultrasonic planar transducer. The results of the projected and experimental fields are compared at various distances for synthetic signals and experimental data. Good correlation is found between the calculated, projected, and experimental data. (C) 1998 Acoustical Society of America.
\end{abstract}

[S0001-4966(98)04109-5]

PACS numbers: 43.20.Px, 43.38.Ar [JEG]

\section{INTRODUCTION}

Several FFT algorithms have been developed that efficiently project harmonic pressure fields. ${ }^{1}$ These approaches allow the time and space dependence of the acoustic pressure in a specific plane to be propagated closer to or away from a source. The use of these methods with experimental data offers a technique for global reproduction of a transducer's field from information obtained at a single plane. Experiments using these methods have been carried out, but have been limited to harmonic cases. $^{2}$

In the present paper, we investigate the projection of radially symmetric acoustic space-time fields using FFT methods. The reconstruction and projection algorithm, similar to that used by Forbes et al. ${ }^{3}$ for simulated space-time fields, is described in Sec. I. Our description optimizes the method for the particular case of axisymmetric field data obtained from an optical sensor. This symmetric field approximation is a reasonable assumption for a quality axisymmetric source and results in a significant savings in the required data and the computer reconstruction time. As a means of verifying the space-time projection algorithm, it is first applied to simulated fields in Sec. II. A previously developed impulse response method ${ }^{4}$ is used to model acoustic fields from planar ultrasonic transducers that approximate those studied experimentally. Our approach is similar to our earlier studies ${ }^{3}$ and is based on using the velocity of the front surface of the transducer to determine the space-time pressure field. An improved transducer model is incorporated into the present study that includes contributions from the back surface of the piezoceramic transducer. ${ }^{5,6}$

An experiment was performed to verify the use of the space-time projection algorithm on real data obtained from a typical ultrasonic piezoelectric transducer. The experiment, described in Sec. III, consists of scanning an optical fiber along a plane parallel to the face of the transducer and obtaining an array of line integrals of the field in that plane. The space-time pressure field in the measurement plane is reconstructed from the fiber-optic measurements using tomographic methods. Fiber-optic imaging was chosen for its high sensitivity, minimal invasiveness, and its ability to easily measure a field in a fixed plane. The field data are then projected away from the measurement plane using the FFTbased method. Finally, the forward projected fields are compared with experimental data at various distances from the source in Sec. IV.

\section{RECONSTRUCTION AND PROJECTION ALGORITHM}

Consider a general axisymmetric pressure field using the cylindrical coordinate system illustrated in Fig. 1, where $p(\rho, z, t)$ denotes the space-time pressure that satisfies the usual linear wave equation, given by

$$
\nabla^{2} p(\rho, z, t)=\frac{1}{c^{2}} \frac{\partial^{2} p(\rho, z, t)}{\partial t^{2}}
$$

The forward projection problem of interest is defined here as mapping the field information in a reference plane, $p\left(\rho, z_{0}, t\right)$, into another plane, $p(\rho, z, t)$ for $z>z_{0}$.

The Hankel transform pair for the pressure is first introduced as 


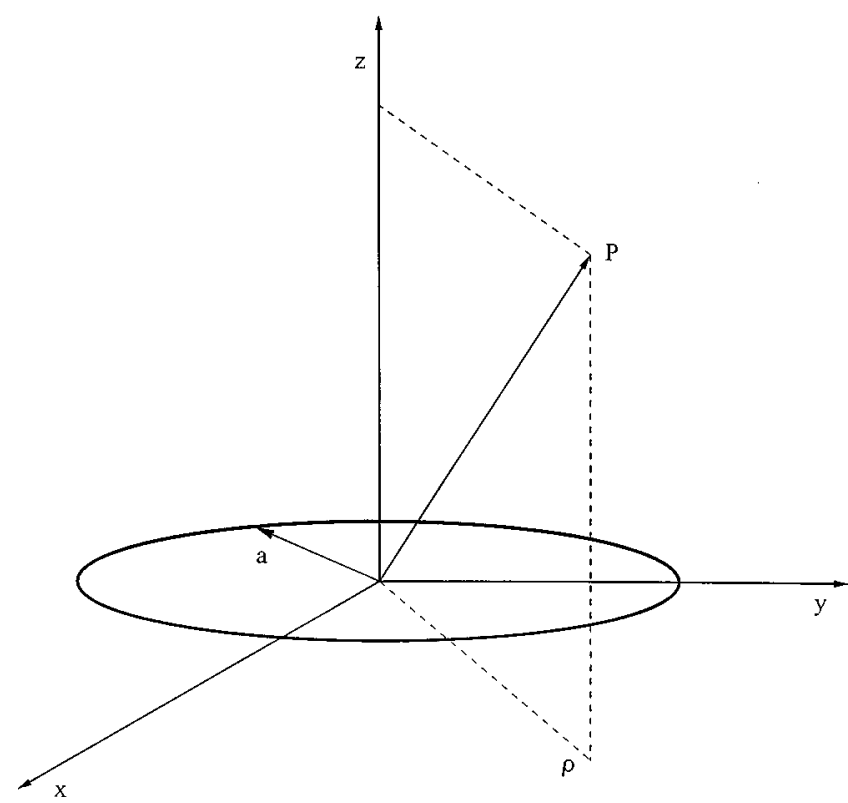

FIG. 1. Circular piston in a planar baffle.

$$
\begin{aligned}
& P(k, z, t)=\int_{0}^{\infty} p(\rho, z, t) J_{0}(k \rho) \rho d \rho, \\
& p(\rho, z, t)=\int_{0}^{\infty} P(k, z, t) J_{0}(k \rho) k d k,
\end{aligned}
$$

$$
P(k, z, t)=\frac{1}{2 \pi} \int_{-\infty}^{\infty} \hat{P}(k, z, \omega) e^{i \omega t} d \omega .
$$

The transform $P(k, z, t)$ is in a wave vector time-space whereas $\hat{P}(k, z, \omega)$ is in a wave vector frequency space. Ob- (a)

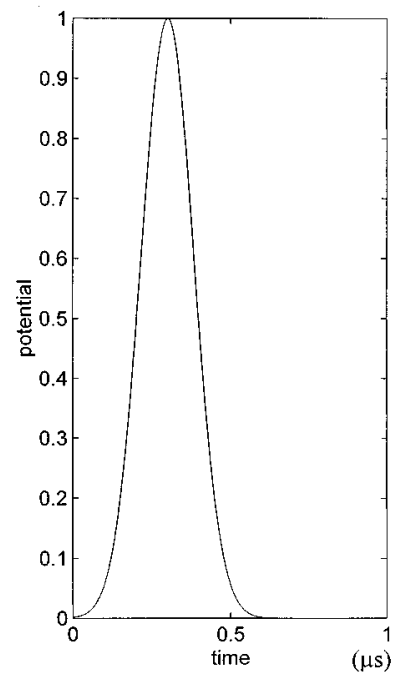

(b)

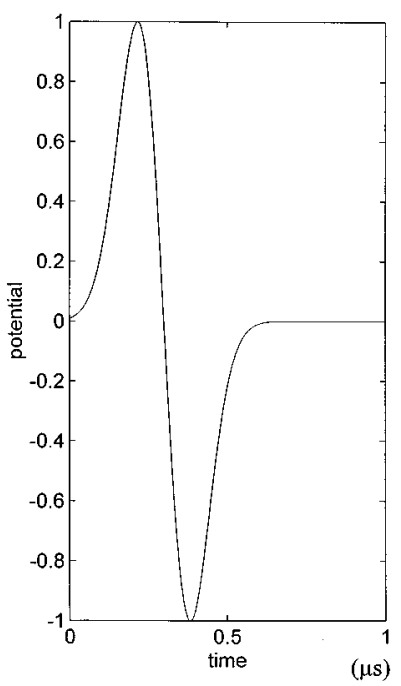

FIG. 2. Driving signal of the field. (a) Voltage applied to transducer. (b) Velocity of transducer face versus time. viously both transforms can be combined but are separated here for later convenience.

Before continuing to the forward projection problem, it is first noted that $P\left(k, z_{0}, t\right)$ can also be evaluated in an indirect manner without an a priori knowledge of $p\left(\rho, z_{0}, t\right)$. To this end, the projection of the pressure field along the $y$-direction in Cartesian coordinates in the plane $z=z_{0}$ is introduced as follows:

$$
g\left(x, z_{0}, t\right)=\int_{-\infty}^{\infty} p\left(x, y, z_{0}, t\right) d y .
$$

The Fourier transform of $g\left(x, z_{0}, t\right)$ with respect to $x$ is then defined as

$$
\tilde{g}\left(k, z_{0}, t\right)=\int_{-\infty}^{\infty} g\left(x, z_{0}, t\right) e^{-i k x} d k .
$$

Finally, it is noted from the projection-slice theorem of Fourier transforms that

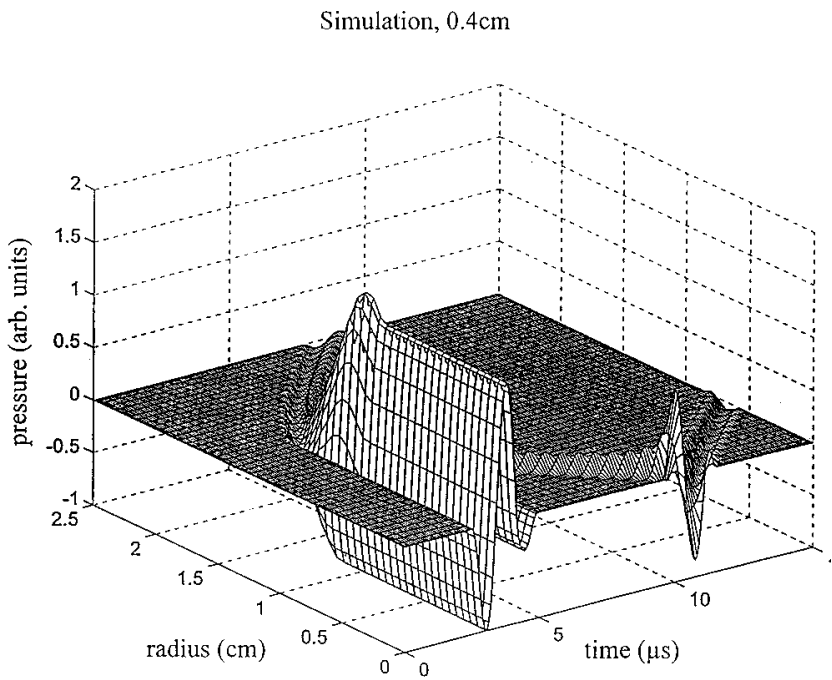

(a)

Projection of Simulation: $0.4 \mathrm{~cm}$ to $0.4 \mathrm{~cm}$

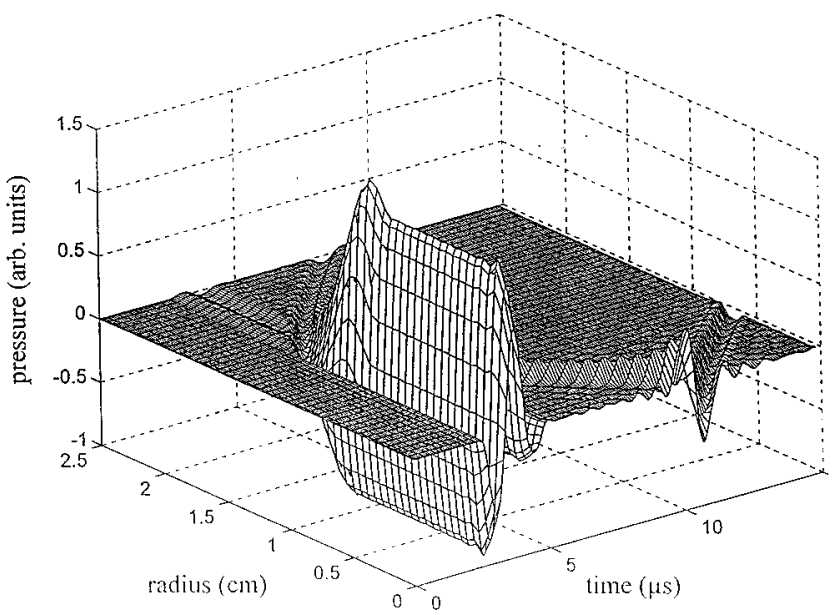

(b)

FIG. 3. Pressure field in the plane $z=0.4 \mathrm{~cm}$ from the source. (a) Impulse response calculation as a function of radial position and time. (b) Projection from $4 \mathrm{~mm}$ onto itself as a function of radial position and time. 


$$
P\left(k, z_{0}, t\right)=\frac{\tilde{g}\left(k, z_{0}, t\right)}{2 \pi} .
$$

Although $p\left(\rho, z_{0}, t\right)$ can be reconstructed from $P\left(k, z_{0}, t\right)$ via the use of Eq. (2), the forward projection of the field can be accomplished directly from $P\left(k, z_{0}, t\right)$ as shown in the following development.

Consider now the forward projection problem of mapping the field information in the reference plane, $p\left(\rho, z_{0}, t\right)$, into $p(\rho, z, t)$ for $z>z_{0}$. From the Fourier-Hankel transform of Eq. (1), it is easily shown that:

$$
\frac{d^{2}}{d z^{2}} \hat{P}(k, z, \omega)+\left(\frac{\omega^{2}}{c^{2}}-k^{2}\right) \hat{P}(k, z, \omega)=0 .
$$

Now Eq. (7) has the following solution for the forward projection problem:

$$
\hat{P}(k, z, \omega)=\hat{P}\left(k, z_{0}, \omega\right) e^{-i \sqrt{\omega^{2} / c^{2}-k^{2}}\left(z-z_{0}\right)},
$$

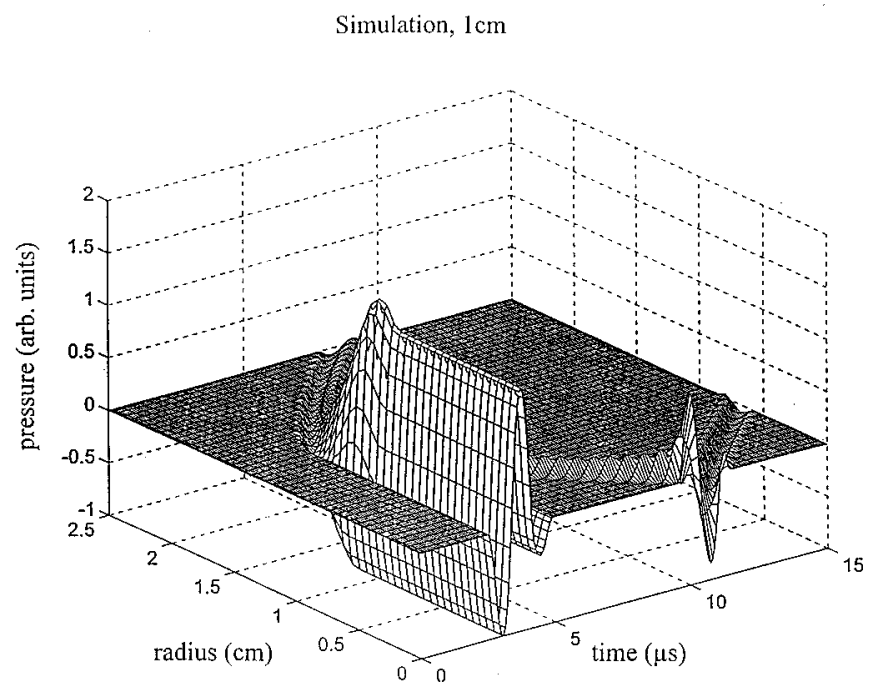

(a)

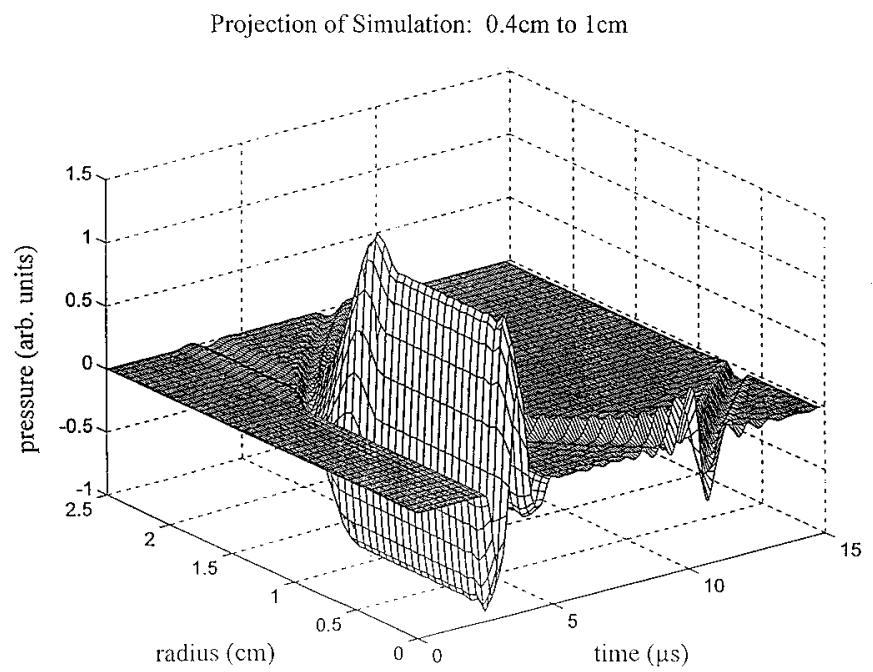

(b)

FIG. 4. Pressure field in the plane $z=1 \mathrm{~cm}$ from the source. (a) Impulse response calculation as a function of radial position and time. (b) Projection from $4 \mathrm{~mm}$ to $1 \mathrm{~cm}$ as a function of radial position and time. where $\hat{P}\left(k, z_{0}, \omega\right)$ is to be determined. But $\hat{P}\left(k, z_{0}, \omega\right)$ can be readily obtained either from the field at $z_{0}$ and Eqs. (2) and (3), in which case it is given by

$$
\hat{P}\left(k, z_{0}, \omega\right)=\int_{0}^{\infty} \int_{0}^{\infty} p\left(\rho, z_{0}, t\right) J_{0}(k \rho) e^{i \omega t} \rho d \rho d t
$$

or from the lateral projection of the field and Eqs. (3) and (6), in which case it is given by

$$
\hat{P}\left(k, z_{0}, \omega\right)=\frac{1}{2 \pi} \int_{-\infty}^{\infty} \widetilde{g}\left(k, z_{0}, t\right) e^{i \omega t} d t .
$$

Finally, from Eqs. (2), (3), and (8), the field at $z$ can be expressed as

$$
\begin{aligned}
p(\rho, z, t)=\frac{1}{2 \pi} & \int_{0}^{\infty} \int_{-\infty}^{\infty} \hat{P}\left(k, z_{0}, \omega\right) \\
& \quad \times e^{-i\left(z-z_{0}\right) \sqrt{\omega^{2} / c^{2}-k^{2}}} J_{0}(k \rho) e^{i \omega t} d k d \omega .
\end{aligned}
$$

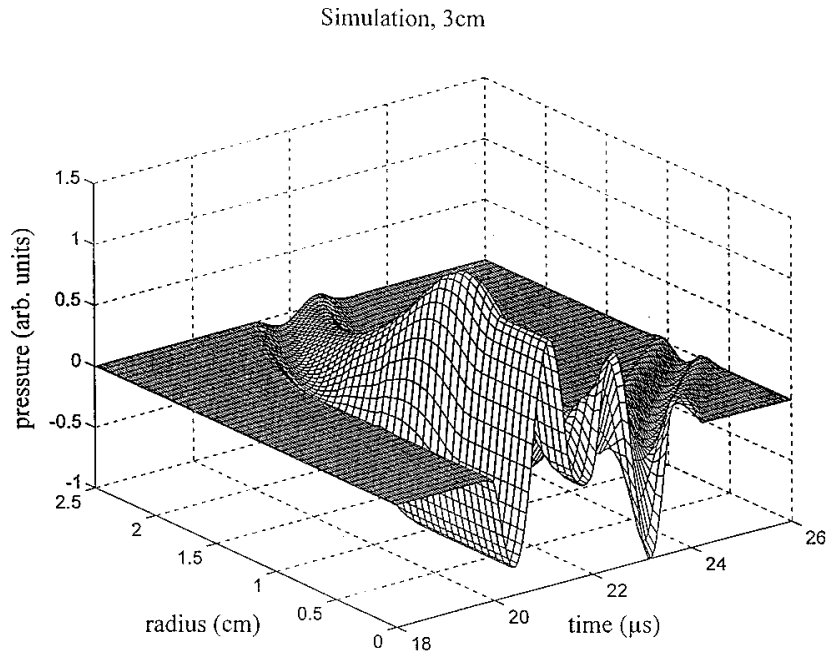

(a)

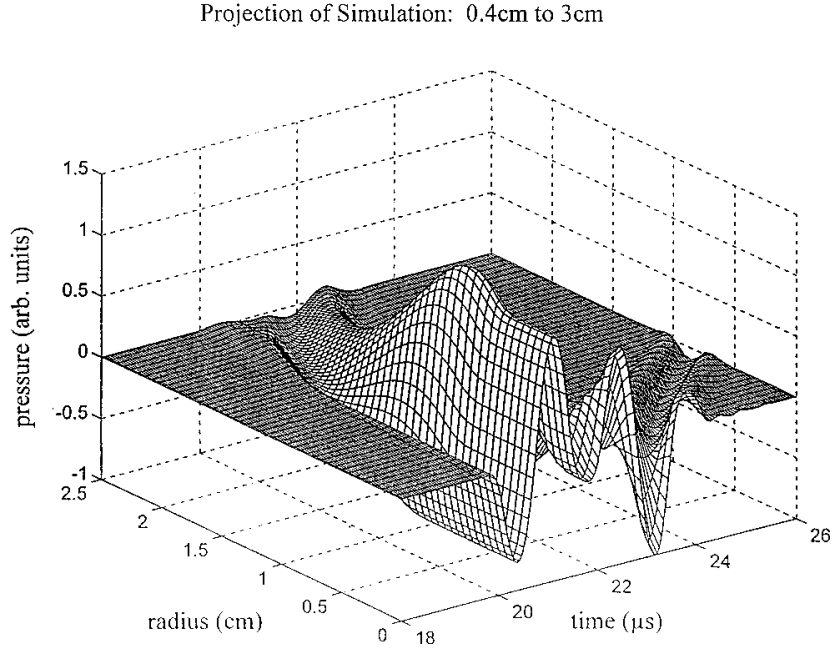

(b)

FIG. 5. Pressure field in the plane $z=3 \mathrm{~cm}$ from the source. (a) Impulse response calculation as a function of radial position and time. (b) Projection from $4 \mathrm{~mm}$ to $3 \mathrm{~cm}$ as a function of radial position and time. 


\section{SIMULATED FIELD OF AN ULTRASONIC TRANSDUCER}

Consider now the space-time pressure field which is generated by a circular piston-type ultrasonic transducer. It is well known that the pressure at a field point $(\rho, z)$ may be expressed as the time derivative of the time convolution of the transducer velocity function $v(t)$ and the impulse response function, $h(\rho, z, t),{ }^{4}$ i.e.,

$$
p(\rho, z, t)=\frac{\partial}{\partial t}[v(t) \otimes h(\rho, z, t)] .
$$

The functional form of the impulse response function depends on whether the radial position of the field point is greater than or less than the size of the transducer.

For a circular transducer of interest, the impulse response function is given by ${ }^{4}$

$$
h= \begin{cases}0 ; & c t<z, \\ \rho c ; & z<c t<\sqrt{z^{2}+(a-\rho)^{2}}, \\ \frac{\rho_{0} c}{\pi} & \arccos \left(\frac{\rho^{2}+(c t)^{2}-a^{2}-z^{2}}{2 \rho \sqrt{(c t)^{2}-z^{2}}}\right) ; \\ & \sqrt{z^{2}+(a-\rho)^{2}}<c t<\sqrt{z^{2}+(a+\rho)^{2}}, \\ 0 ; \quad c t>\sqrt{z^{2}+(a+\rho)^{2}},\end{cases}
$$

for $\rho<a$ and by ${ }^{4}$

$$
h= \begin{cases}0 ; & c t<\sqrt{z^{2}+(a-\rho)^{2}} \\ \frac{\rho_{0} c}{\pi} & \arccos \left(\frac{\rho^{2}+(c t)^{2}-a^{2}-z^{2}}{2 \rho \sqrt{(c t)^{2}-z^{2}}}\right) \\ & \sqrt{z^{2}+(a-\rho)^{2}}<c t<\sqrt{z^{2}+(a+\rho)^{2}} \\ 0 ; & c t>\sqrt{z^{2}+(a+\rho)^{2}}\end{cases}
$$

for $\rho>a$.

The impulse-response method was used to simulate acoustic fields from an ultrasonic transducer with the same excitation, dimensions, and characteristics as that used in the experiment described below. Since the velocity of the front surface of the transducer is required in Eq. (11), $v(t)$ is approximated by starting with a Gaussian signal (full width at half maximum $=2 \mu \mathrm{s}$ ) to represent the applied voltage. The initial velocity at the front surface of the transducer is then represented by the time derivative of the Gaussian. An additional contribution from the back surface of the piezoceramic is included by adding to the front surface velocity a function that has the same shape, but (i) is opposite in phase, (ii) is delayed by the time of flight from the back surface to the front, and (iii) is reduced in amplitude due to radiation into the backing of the piezoelectric disk. ${ }^{7}$ Additional reverberation effects within the disk are neglected. The resultant piston velocity function that was used for the study is shown in Fig. 2(b).

A simulated field was calculated for the plane $z$ $=0.4 \mathrm{~cm}$ from the transducer face. This field was then propagated away from the source using the projection algorithm described in the previous section. Specifically, fields were constructed at planes $0.4,1,3$, and $10 \mathrm{~cm}$ in front of the transducer. The projection to the plane at $0.4 \mathrm{~cm}$ is a forward projection of $0 \mathrm{~cm}$ and is simply used to test the transform algorithms. Next, the impulse response method was used to calculate the field at each of the above distances. The projected and modeled fields are presented in Figs. 3-6 for the initial $z$-value and the four planes of projection. Except for the artifact at early $t$ values for large $r$ when the projection distance is short, the projected and calculated fields match very well.

Direct comparisons of the on-axis and $r=a$ slices of the simulated and projected data are presented in Figs. 7 and 8. The on-axis plots reveal slight oscillations in the projected data for the planes close to the transducer. The edge wave amplitude is also slightly smaller in the projected data. The causes of these discrepancies are discussed below. For planes further away from the transducer and for the slice at $r=a$ the simulated and projected fields agree closely throughout.

As a means of evaluating the effectiveness of our projection algorithm, a quantitative comparison was made between the simulated data and the corresponding projections. An rms measurement of discrepancy between the two was obtained for each distance away from the piston using the quotient

$$
\left[\frac{\sum_{i, j}^{M, N}\left(p_{i j}-p_{i j}^{\prime}\right)^{2}}{\sum_{i, j}^{M, N}\left(p_{i j}\right)^{2}}\right]^{1 / 2},
$$

where $p_{i j}$ is an element of the simulated data and $p_{i j}^{\prime}$ is an element of the field projection. Results range from $11 \%$ at $z=0.4 \mathrm{~cm}$ to $4.0 \%$ at $z=10 \mathrm{~cm}$. The larger error close to the transducer face seems to result from the rapidly varying slopes present in the simulated field. If required, this error could be reduced by increasing the spatial resolution of the data, but at the expense of increased computation time. In addition, the projection algorithm is band limited on both the spatial and temporal axes, causing error in reconstruction of the sharply varying, broad band geometries present in the 1 $\mathrm{cm}$ and $4 \mathrm{~mm}$ simulations. The purpose of this error measurement is only to evaluate only the projection algorithm itself. Similar analysis is thus omitted for the experimental results presented in the following section.

\section{EXPERIMENT PROCEDURES AND RESULTS}

\section{A. Apparatus}

The experimental apparatus is shown in Fig. 9 to consist of a piezoelectric transducer in a water tank, a polarizationmaintaining optical fiber as the sensor, a computer-controlled positioning system for the fiber, and an optical demodulation system. As indicated in the figure, the excitation to the transducer, the positioning of the fiber sensor, and the measurement of the detector output are all computer-controlled (C).

The experiments were conducted using a circular, 2.8cm-diameter, broadband piezoceramic transducer with a peak resonance at $500 \mathrm{kHz}$. As a result of the backing, the Q of the transducer is approximately 2 . The signal to the transducer (T) comes from a Real Time Systems (model 3805) arbitrary waveform generator (AWG) and an ENI power amplifier (PA). The voltage signal applied to the transducer was Gaussian with a peak voltage of $560 \mathrm{~V}$ and a full width at half maximum of $2 \mu \mathrm{s}$. The repetition rate was $1 \mathrm{kHz}$. 


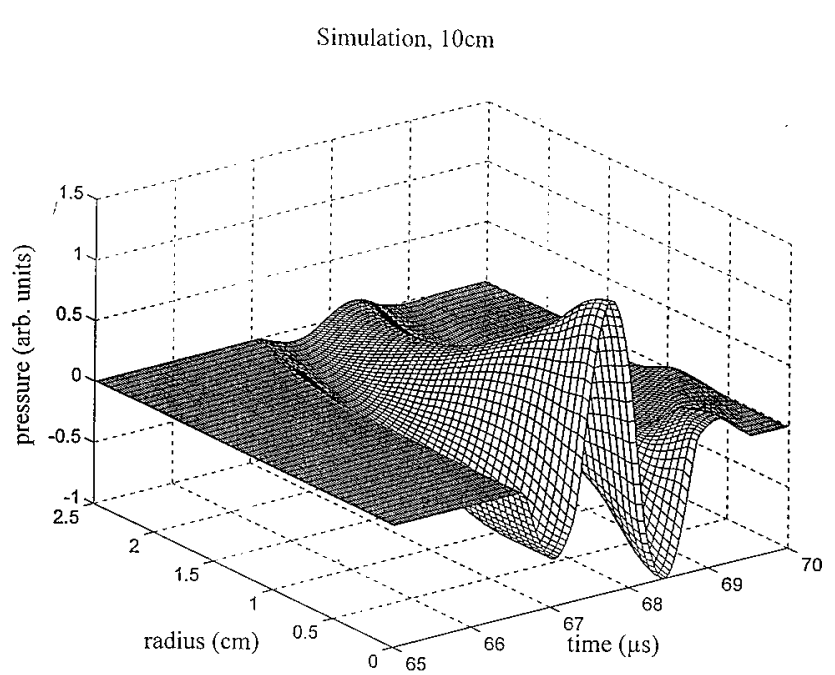

(a)

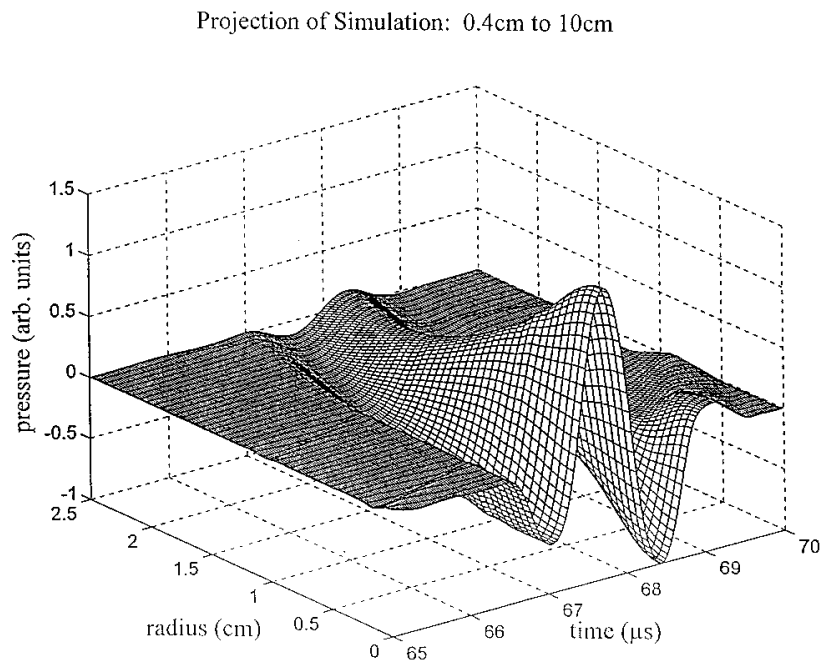

(b)

FIG. 6. Pressure field in the plane $z=10 \mathrm{~cm}$ from the source. (a) Impulse response calculation as a function of radial position and time. (b) Projection from $4 \mathrm{~mm}$ to $10 \mathrm{~cm}$ as a function of radial position and time. (a)

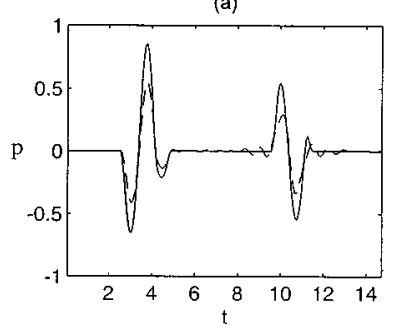

(c)

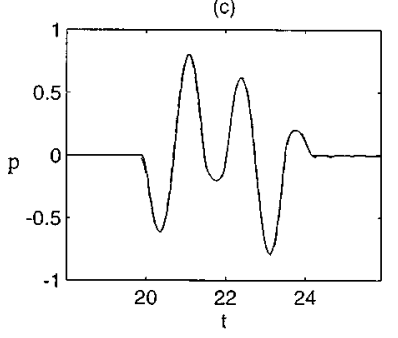

(a)

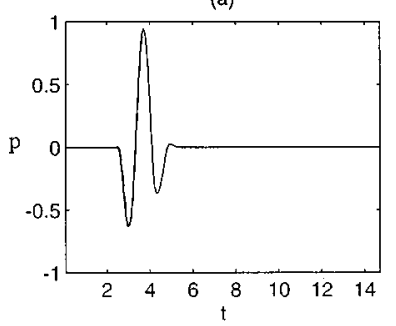

(c)

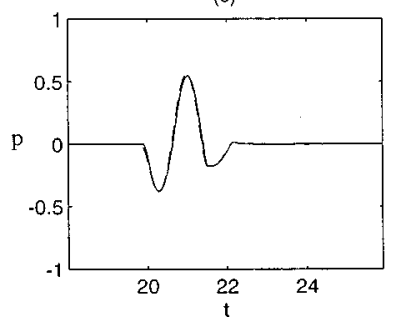

(b)

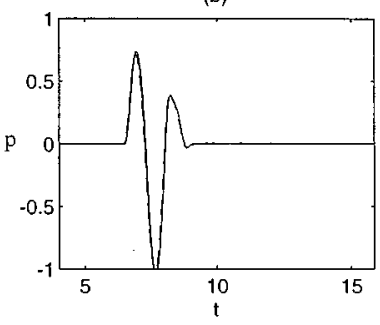

(d)

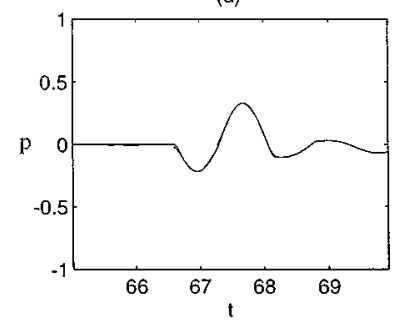

FIG. 8. Simulated pressure (solid) and its projection (dashed) from $0.4 \mathrm{~cm}$ along the radial position $r=a$. (a) $0.4 \mathrm{~cm}$; (b) $1 \mathrm{~cm}$; (c) $3 \mathrm{~cm}$; (d) $10 \mathrm{~cm}$.

The detection system is a fiber-optic polarization interferometer, similar to that described by Chiang et al. ${ }^{8}$ The light beam from a He-Ne laser (L) passes through a polarizer $(\mathrm{P})$ and a quarter-wave plate $(\mathrm{Q})$ to provide circularly polarized light that is focused into the fiber core. Since the polarization-maintaining fiber is inherently birefringent, the polarization state of the light emerging from the fiber is generally elliptical and drifts due to temperature fluctuations. The strain in the fiber from the ultrasonic wave induces additional birefringence that modulates the polarization state of the emerging light beam. ${ }^{9,10}$ A passive quadrature demodulation scheme was used in which the output beam is split (B) and one beam passes through a quarter-wave plate before each of them encounters an analyzing polarizer and a photodetector (D). After taking the root of the sum of the squares of the two signals, the result is independent of the ambient polarization state.

\section{B. Experimental procedures}

At a fixed distance in front of the transducer, the fiberoptic sensor was scanned through the water in $0.15-\mathrm{mm}$ increments, well below the minimum spacing permitted by the sampling theorem. ${ }^{11}$ The time history of the detector output was sampled, with a sampling increment of $0.1 \mathrm{~ms}$, at each

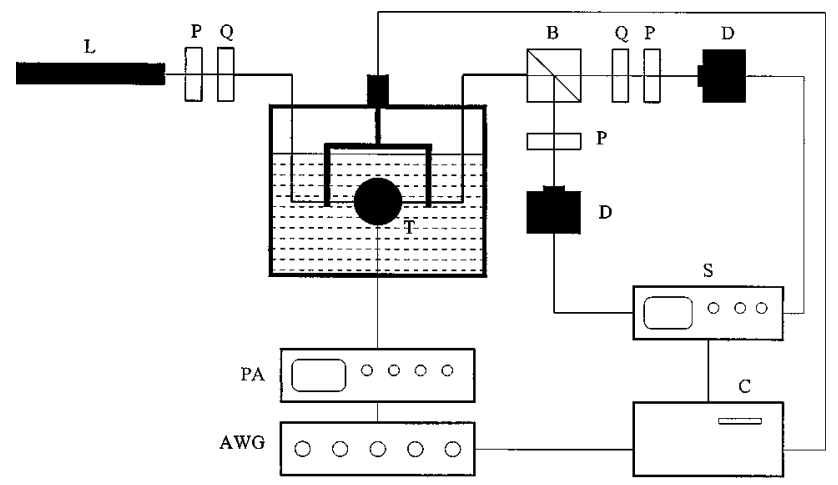

FIG. 9. Experimental setup for fiber-optic acoustic detection.

FIG. 7. On-axis pressure (solid) and its projection (dashed) from $0.4 \mathrm{~cm}$. (a) $0.4 \mathrm{~cm}$; (b) $1 \mathrm{~cm}$; (c) $3 \mathrm{~cm}$; (d) $10 \mathrm{~cm}$. 
position. Due to the integrating effect of the fiber sensor, the line integral of the ultrasonic field, as expressed in Eq. (4), was measured at each position. Data from above and below the piston center were folded such that points equidistant from the axis were averaged. The data were subjected to a low-pass temporal Fourier filter before being tomographically reconstructed. The algorithm used in the reconstruction makes use of the cylindrical symmetry of the field and is expressed in the combination of Eqs. (2), (3), and (9).

\section{Projected data}

Similar to the simulated case, experimental data at 0.4 $\mathrm{cm}$ were projected forward to planes $0.4,1,3$, and $10 \mathrm{~cm}$ from the source. Since statistical error analysis of the fiberoptic sensor has not been performed, verification of the method is presented through a direct graphical comparison of

Experiment, $0.4 \mathrm{~cm}$

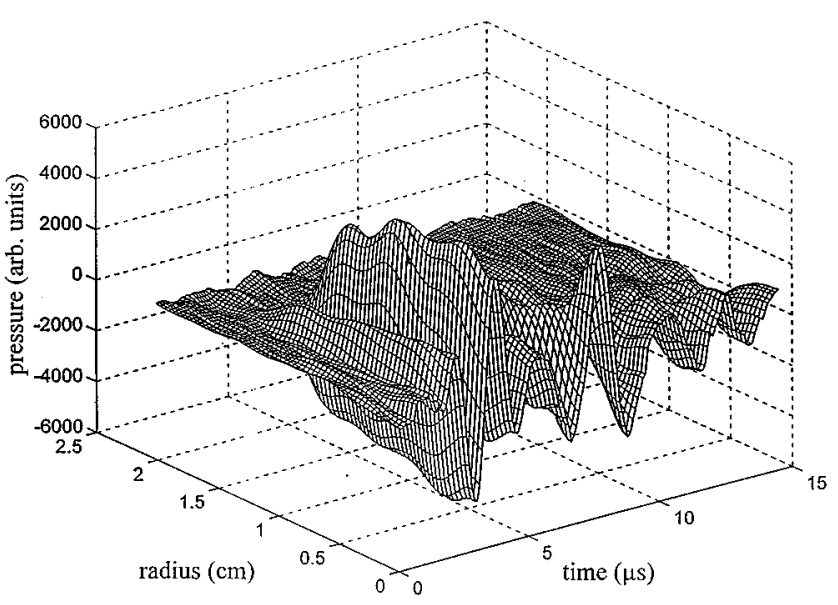

(a)

Projection of Experiment: $0.4 \mathrm{~cm}$ to $0.4 \mathrm{~cm}$

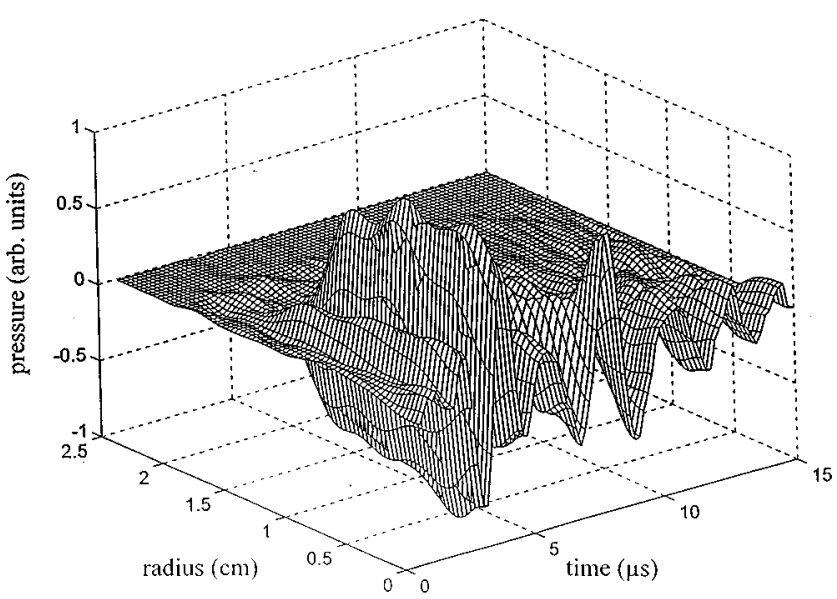

(b)

FIG. 10. Pressure field in the plane $z=0.4 \mathrm{~cm}$ from the source. (a) Experimental result as a function of radial position and time. (b) Projection of experimental result from $0.4 \mathrm{~cm}$. the experimental and projected results. The data were compared with experimental measurements taken at the same distances from the source. Results are presented in Figs. 1013. The projection accurately predicts the major maxima and minima present in the experimental signals.

In Figs. 14 and 15, the time histories for radial slices on-axis and at one piston radius are given. The signals at all distances are found to be in general agreement. A notable exception occurs in the time slice $1 \mathrm{~cm}$ from the piston. On-axis, major peaks are reconstructed, as indicated in Fig. 14(b). However, the projection at one piston radius exhibits an apparent shift in the signal by some fraction of a microsecond. This shift may be a result of experimental error in the projection's originating data set at $0.4 \mathrm{~cm}$. Similarly, the discrepancy in the profile of the projection and experiment along the radial direction at constant times (see Figs. 10-13) is seemingly a result of the combined experimental error from the data used in the projection as well as the data used for comparison at the projected distance. This discrepancy is

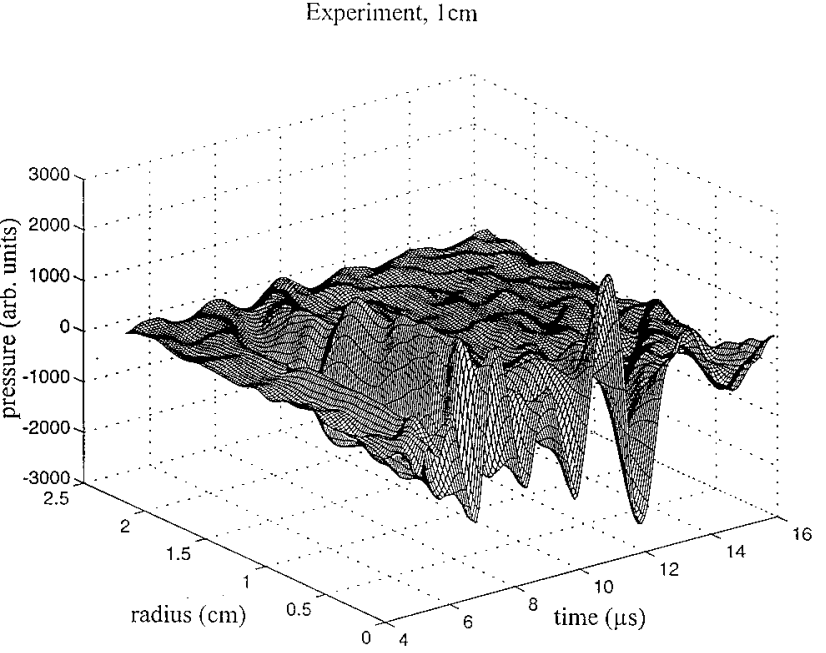

(a)

Projection of Experiment: $0.4 \mathrm{~cm}$ to $1 \mathrm{~cm}$

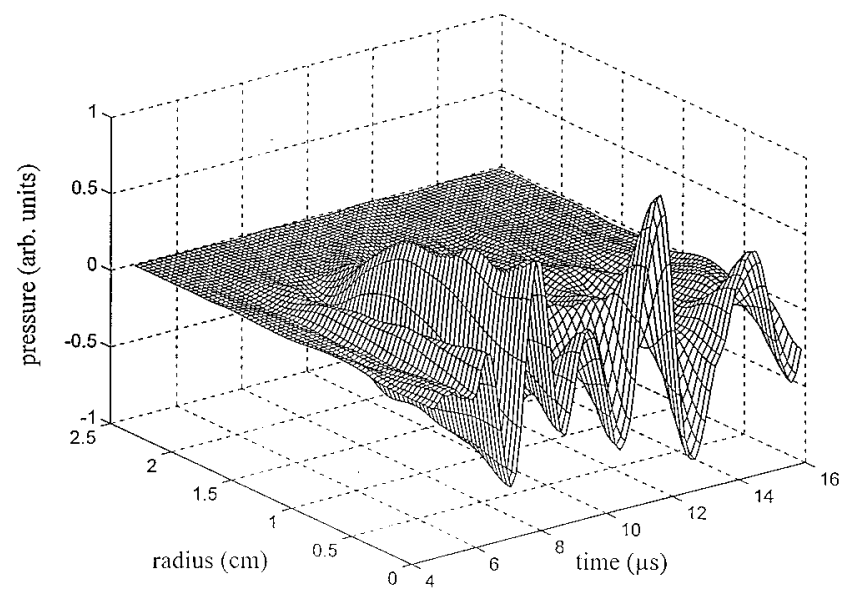

(b)

FIG. 11. Pressure field in the plane $z=1 \mathrm{~cm}$ from the source. (a) Experimental result as a function of radial position and time. (b) Projection of experimental result from $0.4 \mathrm{~cm}$. 


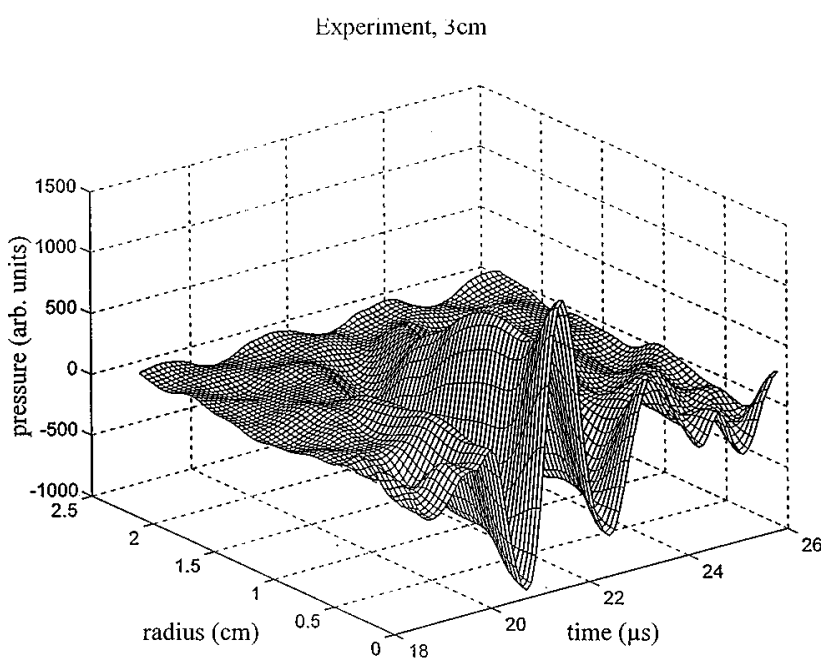

(a)

Projection of Experiment: $0.4 \mathrm{~cm}$ to $3 \mathrm{~cm}$

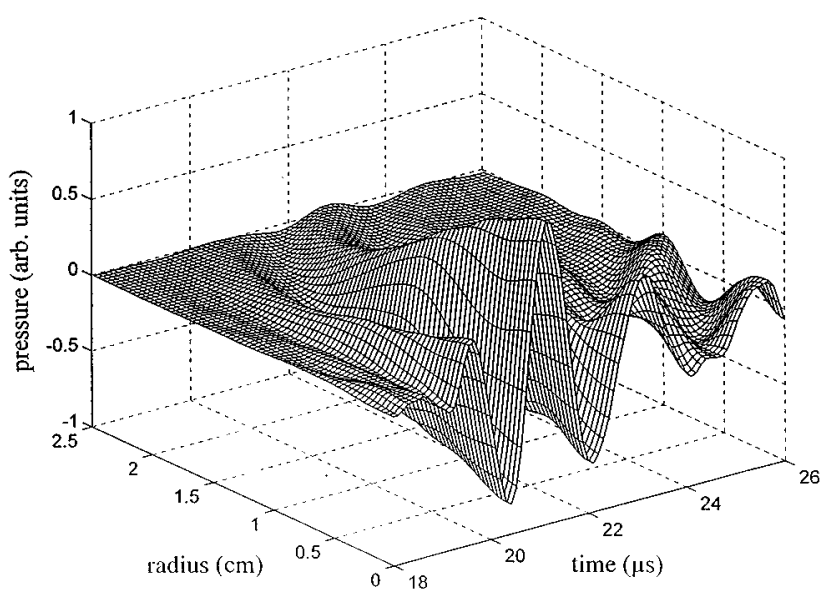

(b)

FIG. 12. Pressure field in the plane $z=3 \mathrm{~cm}$ from the source. (a) Experimental result as a function of radial position and time. (b) Projection of experimental result from $0.4 \mathrm{~cm}$.

most pronounced at $3 \mathrm{~cm}$ (Fig. 12), the projected signal giving a smoother slope. The error is apparently a result of signal information distorted by experimental noise in the 0.4-cm experimental data. Subsequent projection to $3 \mathrm{~cm}$ using experimental data from $1 \mathrm{~cm}$ is in closer agreement with experiment. The overall shape and position of the projected signal, however, agrees with experiment throughout.

\section{SUMMARY}

A projection algorithm is developed for efficiently propagating radially symmetric transient experimental acoustic fields forward in space. Excellent agreement between the modeled fields and their corresponding projections suggests that our projection algorithm is valid for similar experimental signals. To test the algorithm we use tomographically reconstructed data from a fiber-optic detection system. Data in a plane close to the transducer face are projected forward with the Hankel transform based algorithm. Agreement be-
Experiment, $10 \mathrm{~cm}$

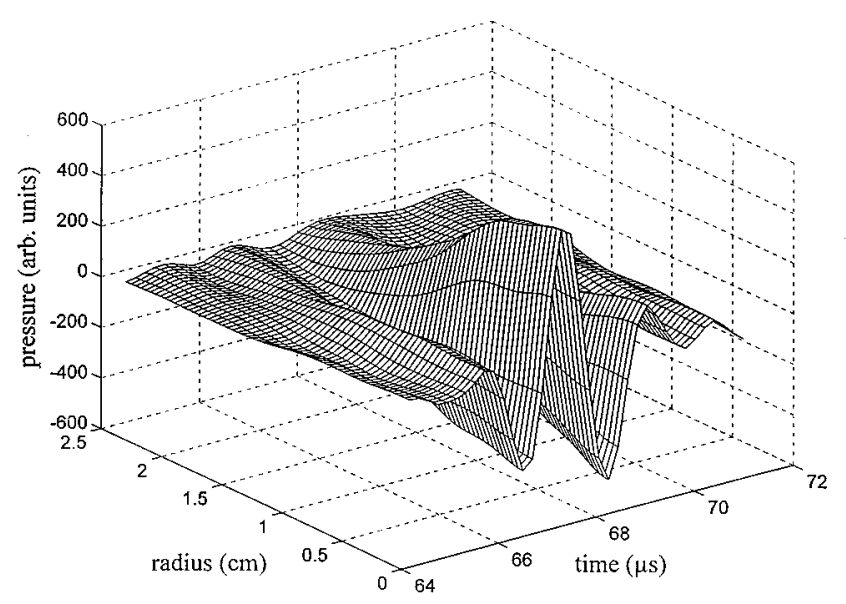

(a)

Projection of Experiment: $0.4 \mathrm{~cm}$ to $10 \mathrm{~cm}$

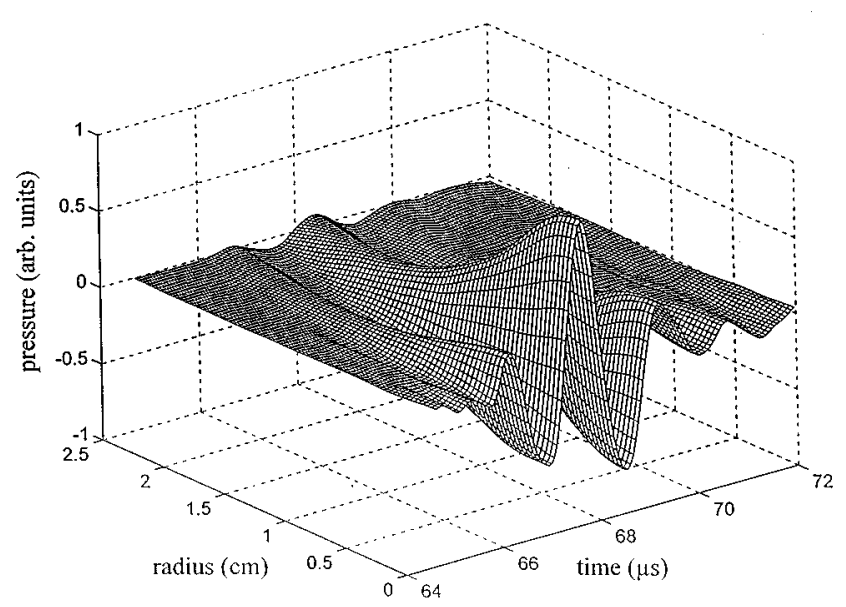

(b)

FIG. 13. Pressure field in the plane $z=10 \mathrm{~cm}$ from the source. (a) Experimental result as a function of radial position and time. (b) Projection of experimental result from $0.4 \mathrm{~cm}$.
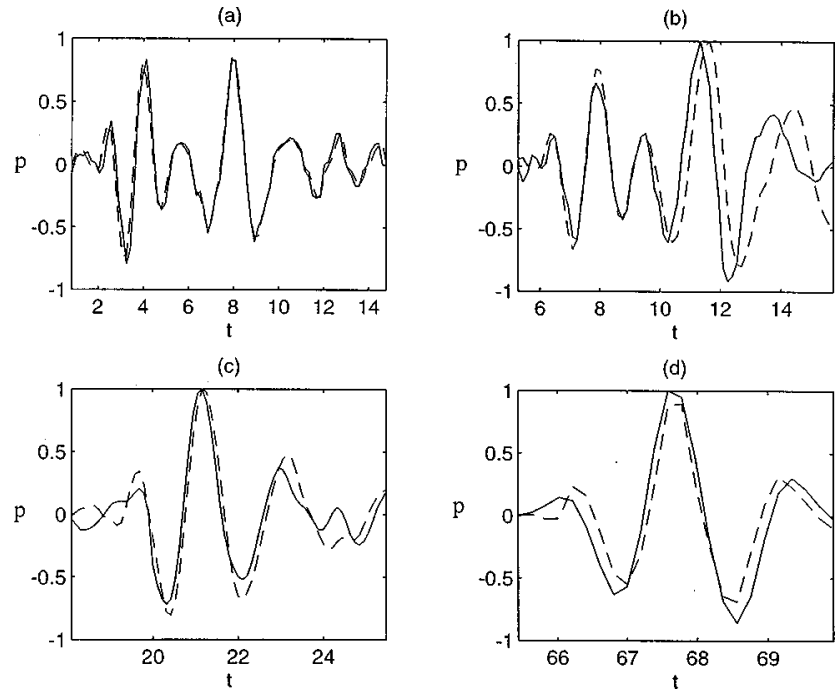

FIG. 14. On-axis experimental pressure (solid) and its projection (dashed) from $0.4 \mathrm{~cm}$. (a) $0.4 \mathrm{~cm}$; (b) $1 \mathrm{~cm}$; (c) $3 \mathrm{~cm}$; (d) $10 \mathrm{~cm}$. 
(a)

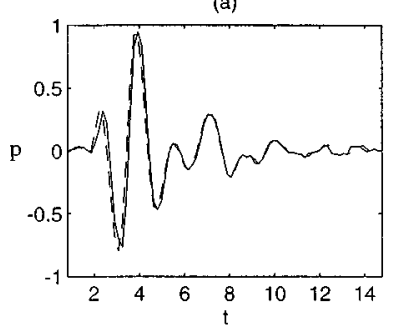

(c)

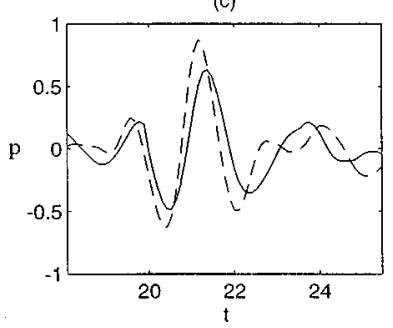

(b)

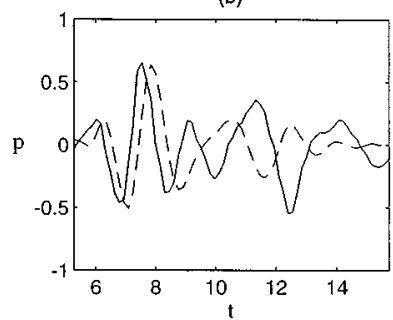

(d)

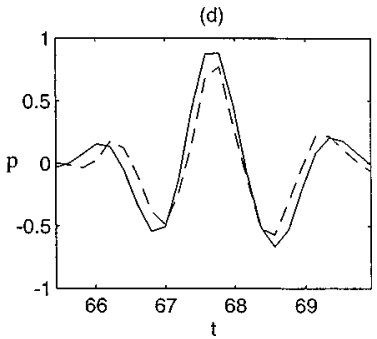

FIG. 15. Experimental pressure (solid) and its projection (dashed) from 0.4 $\mathrm{cm}$ along the radial position $r=a$. (a) $0.4 \mathrm{~cm}$; (b) $1 \mathrm{~cm}$; (c) $3 \mathrm{~cm}$; (d) $10 \mathrm{~cm}$.

tween the experimental data and the associated projected values is readily seen in Figs. 10-13, where major features present in the experimental measurements are also present in the projection, suggesting the feasibility of using the approach for full field characterization of complicated transducer signals. The algorithms employed may be modified for use with arbitrary transducer geometries by generalizing the axisymmetric arguments presented in this paper, although this generalization would entail a significant increase in the required amount of data and computation time. The theory presented is equally valid for backward projections: projecting the field toward the transducer. This technique, however,

requires a careful consideration of evanescent waves, which causes errors in the initial spectrum to increase exponentially as the field is projected backward.

\section{ACKNOWLEDGMENTS}

This work was supported, in part, by the Ocean Technology Center at the University of Rhode Island and by the Office of Naval Research.

${ }^{1}$ P. R. Stepanishen and K. C. Benjamin, "Forward and backward projection of acoustic fields using FFT methods," J. Acoust. Soc. Am. 71, 803-812 (1982)

${ }^{2}$ R. Reibold and F. Holzer, "Complete mapping of ultrasonic fields from optically measured data in a single cross-section," Acustica 58, 11-16 (1985).

${ }^{3}$ M. Forbes, S. V. Letcher, and P. R. Stepanishen, “A wave vector, timedomain method of forward projecting time-dependent pressure fields," $\mathrm{J}$. Acoust. Soc. Am. 90, 2782-93 (1991).

${ }^{4} \mathrm{P}$. R. Stepanishen, "Transient radiation from pistons in an infinite planar baffle,' J. Acoust. Soc. Am. 49, 1629-1638 (1971).

${ }^{5}$ P. R. Stepanishen, "Transient analysis of lumped and distributed parameter systems using an approximate $z$-transform technique,' J. Acoust. Soc. Am. 52, 270-282 (1972).

${ }^{6}$ W. P. Mason, Physical Acoustics (Academic, New York, 1964), Vol. I, Part A.

${ }^{7}$ W. P. Mason, Electromechanical Transducers and Wave Filters (Van Nostrand, New York, 1964).

${ }^{8}$ K. Chiang, H. Chan, and J. Gardner, "Detection of high-frequency ultrasound with a polarization-maintaining fiber," J. Lightwave Technol. 8, 1221-1226 (1990).

${ }^{9}$ R. P. De Paula, L. Flax, J. H. Cole, and J. A. Bucaro, "Single mode fiber ultrasonic sensor,' IEEE J. Quantum Electron. QE-18, 680-683 (1982).

${ }^{10}$ R. P. De Paula, J. H. Cole, and J. A. Bucaro, "Broadband ultrasonic sensor based on induced optical phase shifts in single-mode fibers," J. Lightwave Technol. LT-1, 390-393 (1983).

${ }^{11} \mathrm{~J}$. W. Goodman, Introduction to Fourier Optics (McGraw-Hill, New York, 1968) 